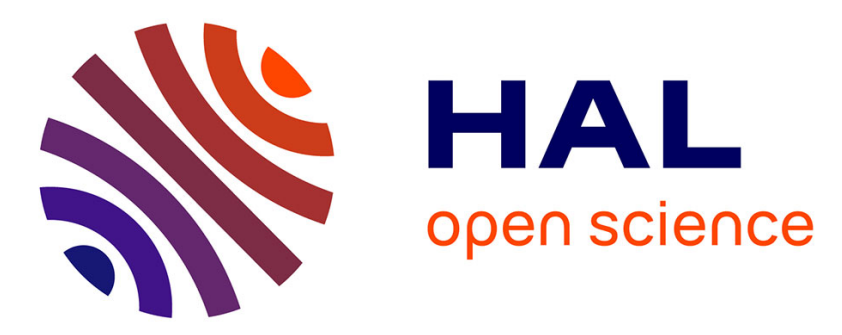

\title{
High resolution spectroscopy of the hydrogen atom. II. Study of line profiles
}

\author{
J.C. Garreau, M. Allegrini, L. Julien, F. Biraben
}

\section{To cite this version:}

J.C. Garreau, M. Allegrini, L. Julien, F. Biraben. High resolution spectroscopy of the hydrogen atom. II. Study of line profiles. Journal de Physique, 1990, 51 (20), pp.2275-2292. 10.1051/jphys:0199000510200227500 . jpa-00212528

\section{HAL Id: jpa-00212528 https://hal.science/jpa-00212528}

Submitted on 1 Jan 1990

HAL is a multi-disciplinary open access archive for the deposit and dissemination of scientific research documents, whether they are published or not. The documents may come from teaching and research institutions in France or abroad, or from public or private research centers.
L'archive ouverte pluridisciplinaire HAL, est destinée au dépôt et à la diffusion de documents scientifiques de niveau recherche, publiés ou non, émanant des établissements d'enseignement et de recherche français ou étrangers, des laboratoires publics ou privés. 
Classification

Physics Abstracts

$35.10-06.20 \mathrm{~J}-32.80 \mathrm{~K}$

\title{
High resolution spectroscopy of the hydrogen atom. II. Study of line profiles
}

\author{
J.C. Garreau (*), M. Allegrini (**), L. Julien and F. Biraben \\ Laboratoire de Spectroscopie Hertzienne de l'ENS( $\left.{ }^{* * *}\right)$, Université Pierre et Marie Curie, 4 place \\ Jussieu, Tour 12, E01 75252 Paris Cedex 05, France
}

(Received 2 February 1990, revised 15 June 1990, accepted 27 June 1990)

\begin{abstract}
Résumé. - Cet article est le deuxième d'une série de trois articles décrivant en détail notre récente mesure de la constante de Rydberg. Nous y présentons le calcul théorique des formes de raies attendues dans notre experience de spectroscopie à deux photons des transitions $2 S-n S$ et $2 S-n D$ de l'atome d'hydrogène. Les différents effets d'élargissement et de déplacement des raies ont été pris en compte, et nous avons calculé la probabilite de transition à deux photons et le déplacement lumineux sous l'effet de l'interaction des atomes metastables $2 S$ avec le laser. Les contributions au signal de toutes les trajectoires atomiques possibles ont été sommées. La comparaison de chaque profil expérimental avec le profil théorique correspondant donne la position de la raie avec une précision de quelques $10^{-11}$.
\end{abstract}

\begin{abstract}
As second part of the detailed description of our recent work on the Rydberg constant measurement, here (paper II) we present our calculations of the line profiles of the hydrogen $2 S-n S$ and 2S- $n$ D Doppler-free two-photon signals observed in our experiment. Various broadening and shift effects have been considered and detailed calculations of the two-photon transition probability and of the light-shift have been done. The contributions of all possible trajectories of the atoms in the metastable beam have been summed to get the detected signal. The fit of each observed line with the corresponding calculated profile provides the line position with a relative accuracy of a few parts in $10^{11}$.
\end{abstract}

\section{Introduction.}

This paper is the second of a series of three (referred to as papers I, II and III) giving a complete description of the method we have used to achieve a determination of the Rydberg constant with a relative accuracy of $1.7 \times 10^{-10}[1]$, limited by the current optical frequency standard.

\footnotetext{
(*) Present address: C.N.E.T., 196 Av. Henri Ravera, 92220 Bagneux, France

$\left({ }^{* *}\right)$ Permanent address: Dipartimento di Fisica, Università di Pisa, Piazza Torricelli 2, 56100 Pisa, Italy.

$\left({ }^{* * *}\right)$ Laboratoire de l'Ecole Normale Supérieure et de l'Université Pierre et Marie Curie, associé au CNRS (URA 18).
} 
Such a high precision measurement needs a very careful study of the observed signal profiles and understanding of physical effects which may broaden or shift the lines.

Our method is to induce the $2 \mathrm{~S}-n \mathrm{D}(n \geq 8)$ Doppler-free two-photon transitions in an atomic beam of hydrogen or deuterium. In paper I [2], we have described the experimental set-up used to produce the metastable atomic beam and to perform the optical excitation in order to observe very narrow signals. In this paper, we discuss the possible causes for the broadening and shift of the lines (sect. 2), we present the detailed calculation of the line profiles (sect. 3) and finally we compare the theoretical curves with the experimental ones (sect. 4).

\section{Broadening and shift of the lines.}

As detailed in reference [2], the 2S- $n$ D Doppler-free two-photon transitions take place in an optical excitation chamber where a metastable atomic beam is collinear with the standing wave obtained by superposition of two counterpropagating laser beams. After a two-photon excitation from the $2 S$ state, about $95 \%$ of atoms in the $n D$ states undergo radiative cascades to the $1 S$ ground state. The two-photon transition can then be detected by observing the corresponding decrease of the metastable beam intensity. A typical recording has been shown in figure 7 of paper I [2] in the case of the 2S-10D two-photon transition in hydrogen. The observed linewidth is about $1 \mathrm{MHz}$ (in terms of the total two-photon transition frequency), to be compared to the natural width of the $10 \mathrm{D}$ level which is $296 \mathrm{kHz}$. Several effects can explain this broadening, the most important being light-shift and saturation. We discuss the various effects in turn.

2.1 LASER LINEWIDTH. - The jitter of the dye laser has been reduced to about $50 \mathrm{kHz}$ [2] and therefore the laser linewidth is responsible for a line broadening of $100 \mathrm{kHz}$.

2.2 FINITE TRANSIT TIME. - The transit time of the atoms through the laser beams is very long because the laser beams and the metastable beam are collinear. In the interaction region, the metastable beam is defined by two diaphragms $7 \mathrm{~mm}$ in diameter and $956 \mathrm{~mm}$ apart as represented in figure 1. For an atomic trajectory making an angle $\theta$ with the laser beam the line broadening [3] is $2 v \sin \theta \sqrt{\ln 2} / \pi w_{0}$ (total width at the half maximum) where $v$ is the atomic velocity (about $3 \mathrm{~km} / \mathrm{s}$ for hydrogen) and $w_{0}$ the beam radius (about $0.6 \mathrm{~mm}$ ). For the largest possible angle of atomic trajectories with respect to the laser beam, the line broadening due to the finite transit time is $19 \mathrm{kHz}$.

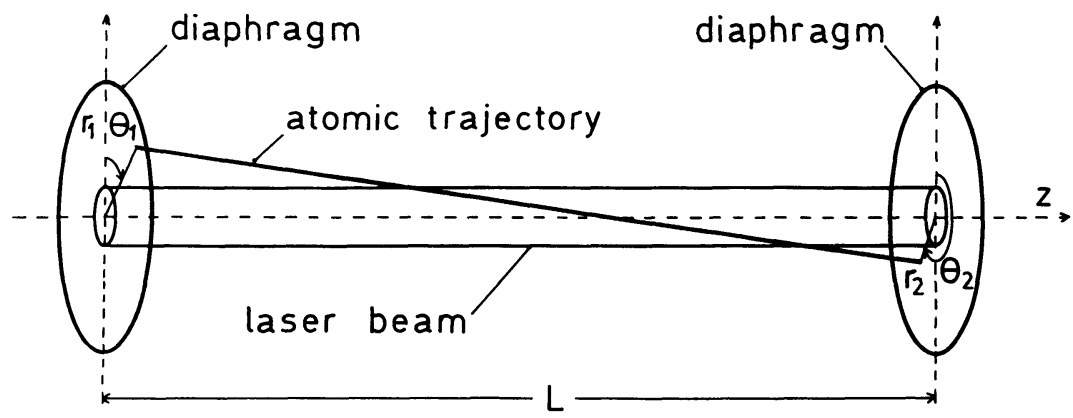

Fig. 1. - Schematic view of atom trajectories in the laser-atom interaction region. 
2.3 SECOND-ORDER DOPPLER EFFECT. - The second-order Doppler effect decreases the line frequency by the quantity $\delta=\frac{v^{2}}{2 c^{2}} \nu_{i f}$ where $\nu_{i f}$ is the atomic frequency. In order to estimate precisely this effect, we have measured the velocity distribution $f(v)$ of the metastable atoms in the beam by looking at the Doppler profile of the Balmer- $\alpha$ line [4] . In a metastable atomic beam the velocity distribution should vary as $v^{4} \exp \left(-v^{2} / 2 \sigma^{2}\right)$ where $\sigma$ is related to the beam temperature $\sigma=(k T / M)^{1 / 2}[5]$. We have measured

$\sigma=1525 \pm 10 \mathrm{~m} / \mathrm{s}$ for hydrogen atoms

$\sigma=983 \pm 10 \mathrm{~m} / \mathrm{s}$ for deuterium atoms.

Since the two-photon transition probability is proportional to the transit time $L / v$ of the atom in the atomic beam ( $L$ is the atomic beam length), we can deduce a first estimate of the mean second-order Doppler shift $\bar{\delta}$ of our lines :

$$
\bar{\delta}=\frac{\nu_{i f} \int_{0}^{\infty} v^{5} \exp \left(-v^{2} / 2 \sigma^{2}\right) d v}{2 c^{2} \int_{0}^{\infty} v^{3} \exp \left(-v^{2} / 2 \sigma^{2}\right) d v}=\frac{2 \sigma^{2} \nu_{i f}}{c^{2}}
$$

For the 2S-10D transition, the mean decrease of the line frequency due to this effect is :

$$
\begin{aligned}
& \bar{\delta}=40.8 \mathrm{kHz} \text { for hydrogen atoms } \\
& \bar{\delta}=17.0 \mathrm{kHz} \text { for deuterium atoms. }
\end{aligned}
$$

Due to the spread of the velocity distribution, there is also a broadening of the same order of magnitude.

2.4 LIGHT-SHIFT AND SATURATION. - In the laser standing wave, the light power may be $50 \mathrm{~W}$ in each propagation direction. For an atom in the center of the laser beam the light-shift is about $560 \mathrm{kHz}$ (case of the 2S-10D transition). The light-shift induces also a broadening due to the variation of the light intensity seen by the atom along its trajectory. For the same light power there is another broadening effect due to the saturation of the two-photon transition. For instance, for an atom travelling exactly along the laser beam axis, the excitation rate is $1.7 \times 10^{5} \mathrm{~s}^{-1}$ while the transit time is $3.3 \times 10^{-4} \mathrm{~s}$. For this trajectory, the saturation is large, while it can be small for trajectories which are parallel to it but offset with respect to the laser beam axis. These two effects are power dependent. Figure 2 shows the variation of the experimental linewidth of the $2 \mathrm{~S}_{1 / 2}-10 \mathrm{D}_{5 / 2}$ transition in deuterium versus the light intensity. It can be seen that the power dependent effects are appreciable, but cannot explain the total linewidth.

To account precisely for the combined effect of natural lifetime, light-shift and saturation we have performed calculations of the line profiles taking into account all possible trajectories of metastable atoms through the laser beams. Details of this treatment are given below in section 3 .

2.5 STARK EFFECT. - The stray electric fields may shift and broaden the line. The shift is due to the quadratic Stark effect and varies as $n^{7}$ ( $n$ is the principal quantum number). The broadening is due to the linear Stark effect and varies as $n^{2}$. Great care has been taken in the experimental set-up to reduce stray electric fields. In order to evaluate their effects, we have excited transitions to higher Rydberg levels which are more sensitive to the Stark effect. Figure 3 shows the signal for the 2S-20D transition. The two fine structure components have the same experimental linewidth $\left(950 \mathrm{kHz}\right.$ ) while the natural width is only $38 \mathrm{kHz}$. For the $20 \mathrm{D}_{3 / 2}$ level (respectively $20 \mathrm{D}_{5 / 2}$ level) the linear Stark splitting between the $\left|m_{J}\right|=3 / 2$ sublevels (respectively $\left|m_{J}\right|=5 / 2$ ) 


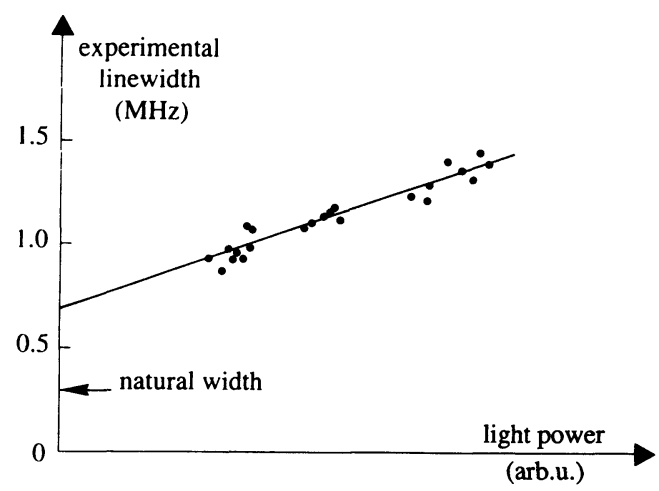

Fig. 2. - Example of the variation of the experimental linewidth as a function of the light power inside the enhancement cavity. Data are relative to the $2 S_{1 / 2}-10 D_{5 / 2}$ transition in deuterium.

is $306 \mathrm{MHz} \mathrm{cm} / \mathrm{V}$ (respectively $217 \mathrm{MHz} \mathrm{cm} / \mathrm{V}$ ) : the Stark broadening is about $50 \%$ larger for the $D_{3 / 2}$ fine structure level than for the $D_{5 / 2}$. In order to obtain an upper limit to the residual electric field, we assume that the linewidth is entirely due to the light-shift broadening (about $700 \mathrm{kHz}$ ) and to the Stark broadening. If we also assume a quadratic sum for these broadenings, we deduce that the Stark broadening is $640 \mathrm{kHz}$ and we obtain an upper limit of about $2 \mathrm{mV} / \mathrm{cm}$. Compared to our preliminary set-up [6], where the stray electric field was $\sim 30 \mathrm{mV} / \mathrm{cm}$, the observed improvement is due to the new vacuum chamber [4] evacuated by two cryogenic pumps. As an example, the effect of a $2 \mathrm{mV} / \mathrm{cm}$ field on the $2 \mathrm{~S}_{1 / 2}-10 \mathrm{D}_{5 / 2}$ transition is a broadening of $\sim 100 \mathrm{kHz}$ and a shift of $\sim 1 \mathrm{kHz}$.

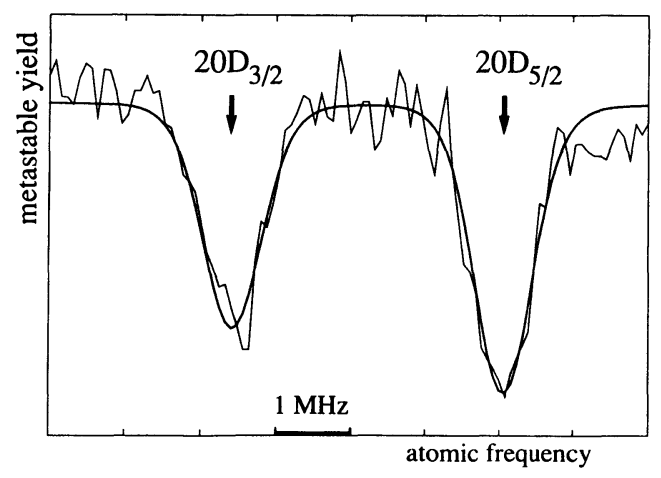

Fig. 3. - Recorded signal showing the fine structure components of the $2 S-20 \mathrm{D}$ transition in deuterium.

2.6 OTHER MINOR EFFECTS. - The contributions of other minor effects are not easily isolated. We have verified that the experimental linewidth is independent of the metastable beam intensity and we think that the effect of collisions with atoms is very small. The Zeeman effect due to the residual magnetic field is more important. The earth magnetic field is compensated using 
three pairs of Helmholtz coils and the residual field is about $20 \mathrm{mG}$. This field induces a Zeeman splitting of about $170 \mathrm{kHz}$ for the $2 \mathrm{~S}_{1 / 2}-n \mathrm{D}_{5 / 2}$ transition.

We now turn to the theoretical calculation of the light-shift and saturation effects, since they are the most important causes of broadening and shift of the lines.

\section{Theoretical lineshapes.}

Atom trajectories in the laser-atom interaction region are sketched in figure 1. Since collisions are negligible in this region, these trajectories are straight lines passing through the two diaphragms. For each trajectory, we have to calculate the survival of metastable atoms through the laser beams. Because of the Gaussian distribution of the light power, an atom travelling along a given trajectory is affected by a varying excitation rate and a varying light-shift. We will first calculate the coefficients of these two effects, and then sum the contributions to the signal of all possible trajectories in the metastable beam. In this calculation we take into account the natural life time, the light-shift and the saturation effect. We neglect the other effects discussed in section 2.

3.1 TwO-PHOTON EXCITATION RATE AND LIGHT-SHIFT. - Consider two atomic states $\mid i>$ and | $f>$ with respective energies $E_{i}$ and $E_{f}\left(E_{f}>E_{i}\right)$. Due to two-photon excitation, the transition rate between these two states is [7] :

$$
\Gamma_{i}=\frac{n^{2} \omega^{2}}{\epsilon_{0}^{2} \hbar^{2}}\left|\sum_{r} \frac{\langle f|\mathrm{~d} . \epsilon| r><r|\mathrm{~d} . \epsilon| i>}{\omega-\omega_{r i}}\right|^{2} \frac{\Gamma_{f}}{\left(2 \omega-\omega_{f i}\right)^{2}+\left(\Gamma_{f} / 2\right)^{2}}
$$

where $n$ is the number of photons per unit volume in each counterpropagating wave, $\omega_{f i}=\left(E_{f}-\right.$ $\left.E_{i}\right) / \hbar, \mid r>$ are all possible intermediate states of energy $E_{r}$ (including the continuum states for which the sum must be replaced by an integral), $\Gamma_{f}$ the natural width of the excited state (we assume that the natural width of the initial state is very small), $\mathbf{d}$ the dipolar momentum operator and $\epsilon$ the polarization vector of the incident light (the two counterpropagating waves have the same polarization).

The excitation rate can be rewritten as:

$$
\Gamma_{i}=\frac{\gamma_{f i} I^{2}}{\left(\Omega-c_{\ell s} I\right)^{2}+\left(\Gamma_{f} / 2\right)^{2}}
$$

In equation (3), frequencies are measured from the null power resonance $\omega_{f i}(0)$, and $\Omega=2 \omega-$ $\omega_{f i}(0)$. The light-shift effect appears explicitly in : $\omega_{f i}=\omega_{f i}(0)+c_{\ell s} I$, where $I$ is the power density related to the photon density $n: I=n \hbar \omega c$ ( $c$ is the velocity of light). The coefficient $\gamma_{f i}$ is :

$$
\gamma_{f i}=\left(\frac{4 \pi a_{0}^{2}}{m c^{2} \alpha}\right)^{2}|\gamma|^{2} \Gamma_{f}
$$

where $a_{0}$ is the Bohr radius, $\alpha$ the fine structure constant, and $\gamma$ is expressed in atomic units $(\hbar=\alpha c=m=1)$. When the two waves are linearly polarised, as in our experiment, $\gamma$ is simply :

$$
\gamma=\sum_{r} \frac{\langle f|z| r><r|z| i>}{\omega-\left(E_{r}-E_{i}\right)} \text { (at.u.) }
$$


From second-order perturbation theory, it turns out that the light- shift coefficient $c_{\ell s}$ is given by :

$$
c_{\ell s}=\left(\frac{4 \pi a_{0}^{2}}{m c^{2} \alpha}\right) \beta
$$

where $\beta$ is $\beta=\beta_{f}-\beta_{i}$

$$
\text { and to } \beta_{n}=\sum_{r}\left[\frac{\mid\langle n|z| r\rangle^{2}}{E_{n}-E_{r}+\omega}+\frac{|\langle n|z| r\rangle|^{2}}{E_{n}-E_{r}-\omega}\right] \quad \text { (at.u.) }
$$

(with $n=i f$ ). In equations (5) and (7), the summation over $\mid r>$ states includes an integral over the continuum states. We have chosen here the r.E gauge rather than the P.A one to describe the electric dipole interaction. Although the final result must be the same, it is known that convergence is better in the r.E gauge [8]. We have performed a numerical summation over the intermediate states $|r\rangle$. The formulas involved in the calculation of the dipole matrix elements are listed in the appendix. We give the $2 \mathrm{~S}-n \mathrm{D}$ and $2 \mathrm{~S}-\mathrm{nS}$ transition amplitudes in table Ia and Ib respectively. The contribution due to the intermediate $3 \mathrm{P}$ state, discrete states and continuum are also given. For the $2 S-n D$ transition amplitude, the major contribution to the sum (5) is due to the sum over the discrete states. In this case the approximation of only one intermediate state (3P) fails by about $30 \%$ (except for the 2S-3D transition). For the $2 \mathrm{~S}-n \mathrm{~S}$ transitions (much weaker than the $2 S-n D$ ones), we find that the continuum gives a dominant contribution and that the approximation of one intermediate state completely fails. Table Ic gives the light-shift coefficients. We find that the $n \mathrm{~S}$ and $n \mathrm{D}$ shifts are very similar. All these results are consistent with a calculation by D. Delande with the Sturmian function method [9] .

Table Ia. - 2S- $n \mathrm{D}$ two-photon transition amplitude $\gamma$ in atomic units : contributions of the various intermediate states.

\begin{tabular}{|c|c|c|c|c|}
\hline$n$ & 3P state & discrete states & continuum & total $\gamma$ \\
\hline 3 & 264.80 & 41.476 & -1.102 & 40.374 \\
4 & -306.29 & -211.31 & -2.23 & -213.54 \\
5 & -160.07 & -163.75 & -3.34 & -167.09 \\
6 & -114.55 & -128.82 & -4.34 & -133.16 \\
7 & -90.00 & -104.65 & -5.22 & -109.86 \\
8 & -75.840 & -86.957 & -5.980 & -92.937 \\
9 & -64.935 & -73.412 & -6.643 & -80.056 \\
10 & -56.584 & -62.700 & -7.220 & -69.920 \\
11 & -49.940 & -54.024 & -7.723 & -61.747 \\
12 & -44.516 & -46.870 & -8.164 & -55.033 \\
15 & -32.960 & -31.481 & -9.196 & -40.677 \\
20 & -22.088 & -16.894 & -10.293 & -27.187 \\
$\infty$ & & & & $-2535.8 n^{-3 / 2}$ \\
\hline
\end{tabular}


Table Ib. - 2S-nS two-photon transition amplitude $\gamma$ in atomic units : contribution of the various intermediate states.

\begin{tabular}{|c|c|c|c|c|}
\hline$n$ & 3P state & discrete states & continuum & total $\gamma$ \\
\hline 3 & 374.45 & 271.37 & -8.30 & 263.07 \\
4 & -110.60 & 27.892 & -11.263 & 16.629 \\
5 & -58.459 & 4.115 & -13.495 & -9.380 \\
6 & -42.094 & 0.442 & -15.154 & -14.711 \\
7 & -33.565 & 0.899 & -16.392 & -15.494 \\
8 & -28.043 & 2.403 & -17.323 & -14.921 \\
9 & -24.050 & 4.104 & -18.028 & -13.925 \\
10 & -20.982 & 5.730 & -18.565 & -12.836 \\
11 & -18.534 & 7.197 & -18.976 & -11.778 \\
12 & -16.533 & 8.489 & -19.289 & -10.800 \\
15 & -12.256 & 11.436 & -19.843 & -8.410 \\
20 & -8.222 & 14.245 & -20.091 & -5.846 \\
$\infty$ & & & & $-572.8 n^{-3 / 2}$ \\
\hline
\end{tabular}

3.2 DESTRUCTION PROBABILITY OF THE METASTABLE ATOMS. - From the light-shift coefficient and the transition rate given above, we are able to deduce the contribution to the signal of a given atom trajectory. Let us consider a straight trajectory (see Fig. 1), starting at the point $\left(r_{1}, \theta_{1}\right)$ on the first diaphragm and ending at the point $\left(r_{2}, \theta_{2}\right)$ on the second diaphragm (the cylindrical symmetry of the problem allows us to put $\theta_{1}=0$ ). The laser intensity along this trajectory varies with the longitudinal component $z$ of the atomic position as :

$$
I(z)=I_{0} \exp \left\{-2[\rho(z) / w(z)]^{2}\right\}
$$

where $\rho$ is the radial distance to the laser beam axis and $w$ the radius of the laser beam (the laser beam is supposed to be perfectly aligned with the two diaphragms). For an atom travelling with velocity $v\left(v_{z}\right.$ is the component of this velocity along the laser beam axis), the probability that a transition has taken place during this trajectory is :

$$
P\left(r_{1}, \theta_{1}, r_{2}, \theta_{2}\right)=1-\exp \left\{\frac{1}{v_{z}} \int_{0}^{L} \mathrm{~d} z \frac{\gamma_{f i}[I(z)]^{2}}{\left[\Omega-c_{\ell s} I(z)\right]^{2}+\left(\Gamma_{f} / 2\right)^{2}}\right\}
$$

In our experiment the spatial distribution of the laser beam intensity is determined by the FabryPerot enhancement cavity, made of two mirrors $1.3 \mathrm{~m}$ apart and with curvature radii of $4 \mathrm{~m}$. The distance between the end mirror of this optical cavity and the first diaphragm defining the metastable beam is $134 \mathrm{~mm}$.

To get equation (9), we have assumed that the laser intensity seen by the atom along its trajectory varies slowly with respect to the lifetime of the excited state. The above treatment has to be corrected to take account of certain other effects, as follows: 
Table Ic. - Light-shift coefficients (in atomic units) $\beta_{i}$ for the $2 S$ level ( $\beta_{i}$ depends on $n$ through the laser frequency $\omega)$, and $\beta_{f}$ for $n \mathrm{~S}$ and $n \mathrm{D}$ levels.

\begin{tabular}{|c|c|c|c|}
\hline$n$ & $2 S$ & $n S$ & $n D$ \\
\hline 3 & -153.35 & -1493.2 & -1377.9 \\
4 & -202.21 & 451.69 & 530.77 \\
5 & -249.37 & 363.35 & 363.69 \\
6 & -290.96 & 324.42 & 316.84 \\
7 & -326.14 & 303.74 & 296.46 \\
8 & -355.31 & 291.35 & 285.43 \\
9 & -379.28 & 283.70 & 278.63 \\
10 & -398.95 & 277.76 & 274.08 \\
11 & -415.14 & 273.78 & 270.85 \\
12 & -428.53 & 270.80 & 268.45 \\
15 & -456.86 & 265.32 & 264.01 \\
20 & -482.11 & 261.18 & 260.58 \\
$\infty$ & -519.17 & 256.0 & 256.0 \\
\hline
\end{tabular}

i) Hyperfine structure of the $2 S$ metastable state. In our experiment we resolve the hyperfine structure of the $2 \mathrm{~S}$ metastable state and we have studied the most intense component, namely the transition $2 \mathrm{~S}_{1 / 2}(F=1) \rightarrow n \mathrm{D}_{5 / 2}$ in hydrogen and the transition $2 \mathrm{~S}_{1 / 2}(F=3 / 2)-n \mathrm{D}_{5 / 2}$ in deuterium. However, our detection method - quenching of all the metastable atoms downstream the atomic beam - does not differentiate between the various hyperfine sublevels. As the metastable atoms are created by electronic bombardment with a large energy spectrum around the 1S-2S transition $(10.2 \mathrm{eV})$, we make the assumption that the hyperfine sublevels of the $2 \mathrm{~S}_{1 / 2}$ are equally excited i.e. with a weight $2 F+1$ ( $F=0$ and 1 in hydrogen, $F=1 / 2$ and $3 / 2$ in deuterium). Thus the transition probability given by equation ( 9 ) for one hyperfine sublevel must be multiplied by a factor $C_{\mathrm{hs}}$ to get the transition probability related to all metastable atoms, independently of the initial hyperfine sublevel. $C_{\mathrm{hs}}$ is the ratio between the population of the hyperfine sublevel in optical resonance with the laser and the total metastable population:

$$
C_{\mathrm{hs}}=3 / 4 \text { in hydrogen }
$$
and $C_{\mathrm{hs}}=2 / 3$ in deuterium.

ii) Fine structure of the excited level. The fine structure of the excited level involved in the transitions observed in our experiment is also resolved and we have only studied the most intense component corresponding to $J=5 / 2$. As the two-photon transition probability is proportional to the degeneracy $2 J+1$ of the fine structure sublevel, we have to multiply the coefficient $\gamma_{f i}$ in equation (9) by the factor 0.6 .

iii) Repopulation of the $2 S$ state. After optical excitation, the $n \mathrm{D}$ states undergo radiative cascades to the $1 \mathrm{~S}$ state or to the $2 \mathrm{~S}$ state, as shown in figure 4 . Table II gives the probability $R_{n}$ of a radiative cascade to the $2 S$ state, calculated taking into account only two-photon cascades. We 
Table II. - Two-photon cascade probability from $n D$ to $2 S$ level.

\begin{tabular}{|c|c|}
\hline$n$ & $R_{n}$ \\
\hline 8 & 0.0513 \\
10 & 0.0536 \\
12 & 0.0548 \\
15 & 0.0558 \\
20 & 0.0567 \\
$\infty$ & 0.0582 \\
\hline
\end{tabular}

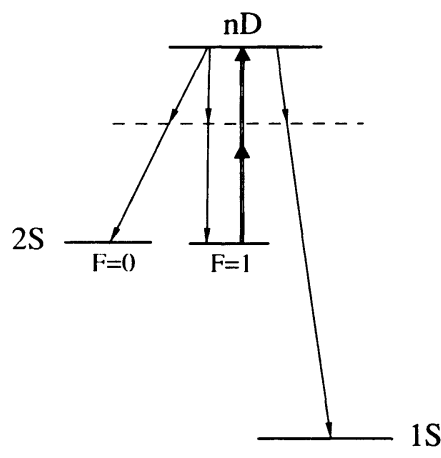

Fig. 4. - Various pathways for two-photon radiative cascade from the laser excited $n \mathrm{D}$ level.

have verified that the effect of the four-photon radiative cascades is only $~ 5 \%$ of the preceding one [10] .

It is also necessary to take into account the effect of the $2 \mathrm{~S}$ state hyperfine pumping : after the two-photon excitation and the radiative cascade, the atom may be in the $F=0$ or $F=1$ hyperfine sublevel of the $2 \mathrm{~S}$ state with the probabilities $p_{0}$ and $p_{1}$ (respectively $p_{1 / 2}$ and $p_{3 / 2}$ for deuterium). When the two-photon excitation is made from the $F=1$ sublevel $(F=3 / 2$ for deuterium) we have calculated [10]:
$p_{0}=2 / 15 R_{n} \quad p_{1}=13 / 15 R_{n}$
for hydrogen
$p_{1 / 2}=8 / 45 R_{n} \quad p_{3 / 2}=37 / 45 R_{n}$
for deuterium.

To take into account this pumping effect, we have solved a system of rate equations involving the two hyperfine sublevels of the $2 S$ state and the excited state [10]. The final result for the destruction probability for a metastable atom at the end of its trajectory is :

$$
\begin{array}{r}
P^{\prime}\left(r_{1}, \theta_{1}, r_{2}, \theta_{2}\right)=\left(1-\frac{p_{0}}{1-p_{1}}\right) C_{\mathrm{hs}} \times \\
\times\left[1-\exp \left\{-\frac{1}{v_{z}} \int_{0}^{L} d z \frac{0.6\left(1-p_{1}\right) \gamma_{f i}[I(z)]^{2}}{\left[\Omega-c_{\ell \mathrm{s}} I(z)\right]^{2}+\left(\Gamma_{f} / 2\right)^{2}}\right\}\right]
\end{array}
$$


The first factor of equation (10) describes the optical pumping from the $F=1$ hyperfine sublevel to the $F=0$ one. The factor $0.6\left(1-p_{1}\right)$ takes into account the fine structure of the $\mathrm{nD}$ state and the inefficiency of the two-photon process when the atom radiates to the initial $F=1$ sublevel. For deuterium $p_{0}$ and $p_{1}$ have to be replaced by $p_{1 / 2}$ and $p_{3 / 2}$ respectively.

3.3 STATISTICS OF TRAJECTORIES AND RESULTS. - To calculate the signal due to all the metastable atoms, we would have to sum equation (10) over all velocities and over all possible trajectories. In fact we have not performed the summation over the actual velocity distribution but have made the calculation with a mean velocity $v_{\mathrm{m}}$ :

$$
v_{\mathrm{m}}=\left\langle 1 / v>^{-1}=\frac{3}{2} \sqrt{\frac{\pi}{2}} \sigma\right.
$$

This approximation is valid in the limit of low laser intensity when it is possible to expand the exponential term of equation (10). We have tested this approximation by making the summation over the velocity distribution in a particular case. For a power of $50 \mathrm{~W}$, the difference between the lineshape obtained with the exact velocity distribution and the one calculated with the mean velocity is about $3 \%$. We have also evaluated the effect of the second-order Doppler effect. In this case the term $\Omega-c_{\ell s} I(z)$ has to be replaced in equation (10) by :

$$
\Omega-c_{\ell s} I(z)+\omega_{f i} v^{2} / 2 c^{2}
$$

In the case of the 2S-10D transition we obtain a global second-order Doppler shift of $-40.6 \mathrm{kHz}$ for hydrogen and $-16.5 \mathrm{kHz}$ for deuterium [10] . These values are in very good agreement with the result of the approximation made in section $2.3(-40.8 \mathrm{kHz}$ and $-17.0 \mathrm{kHz}$ respectively). For these reasons, we have performed the calculations with the mean velocity and, in order to take into account the second-order Doppler effect, we have corrected the line position for the quantity given in equation (1).

To sum over all possible trajectories, it is necessary to know their spatial distribution. The recoil involved in electronic excitation causes the metastable trajectories to have a large dispersion around a mean direction [4] . For this reason we assume a uniform distribution for the impacts of all possible trajectories on the surface of the diaphragm ending the optical interaction region. However, at the beginning of the metastable atomic beam, the spatial distribution of the trajectories is probably more complicated, because it is determined by the various characteristics of the electronic excitation (for instance the spatial distribution of the stray quenching electric field or the spatial distribution of the electronic density). We have then approximated this unknown spatial distribution by an uniform one on a virtual diaphragm centered on the real diaphragm at the beginning of the atomic beam but with a smaller radius $R_{1}$. Finally the lineshape is given by:

$$
\mathcal{P}=\frac{4}{\pi R_{1}^{2} R_{2}^{2}} \int_{0}^{R_{1}} \mathrm{~d} r_{1} r_{1} \int_{0}^{R_{2}} \mathrm{~d} r_{2} r_{2} \int_{0}^{\pi} \mathrm{d} \theta_{2} P^{\prime}\left(r_{1}, 0, r_{2}, \theta_{2}\right)
$$

We have assumed that we have a cylindrical symmetry and that the laser beam is well aligned with respect to the atomic beam. The radius $R_{2}$ is the radius of the real diaphragm at the end of the atomic beam $\left(R_{2}=3.5 \mathrm{~mm}\right)$. As explained below, the value $R_{1}=1.5 \mathrm{~mm}$ was selected by comparison of the theoretical curves with the experimental ones.

A set of theoretical curves obtained for various light power is shown in figure 5 in the case of the $2 S_{1 / 2}-10 D_{5 / 2}$ transition in deuterium. The lines are clearly blue-shifted and show an asymmetry 


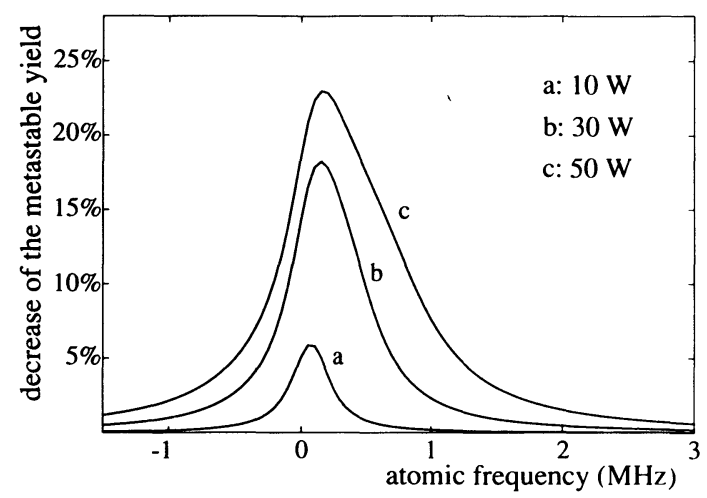

Fig. 5. - Theoretical lineshapes for the $2 S_{1 / 2}-10 D_{5 / 2}$ transition in deuterium.

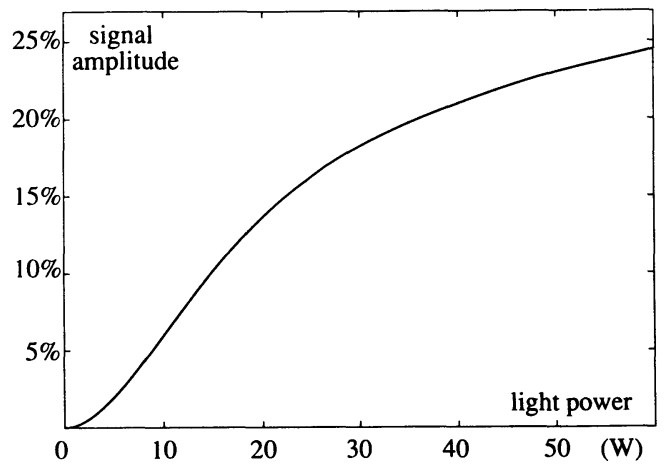

Fig. 6. - Theoretical line intensity $v$ s. light power for the same transition as in figure 5.

for high power. There is also a significant broadening. The line intensity varies from $6 \%$ at $10 \mathrm{~W}$ to $23 \%$ at $50 \mathrm{~W}$. There is a strong saturation which is illustrated in figure 6 . In figure 7 we have plotted the line position (center at the half-maximum) versus the light power. The apparent lightshift is slightly non-linear, due to the saturation of the two-photon excitation probability.

\section{Study of the experimental line profiles.}

4.1 FIT PROCEDURE. - Our purpose is to determine very precisely the transition frequency without light-shift with respect to our very stable reference cavity. With this aim it is necessary to determine the light power seen by the atoms.

As explained in paper I, we detect the two-photon transition by measuring the decrease of the metastable beam intensity versus the laser frequency. For this reason we can calibrate this decrease with respect to the metastable yield measured when the laser is off resonance. The basic idea of the comparison between the theoretical curves and the experimental ones is to use this 


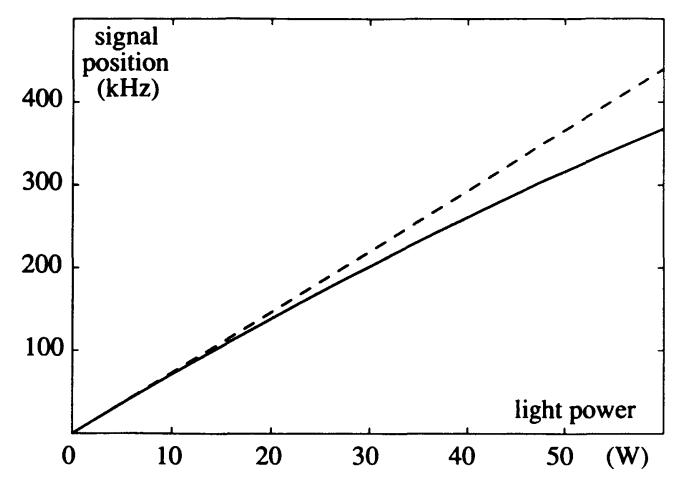

Fig. 7. - Theoretical light-shift vs. light power (same transition as Fig. 5 and 6). The dotted line indicates a linear light shift.

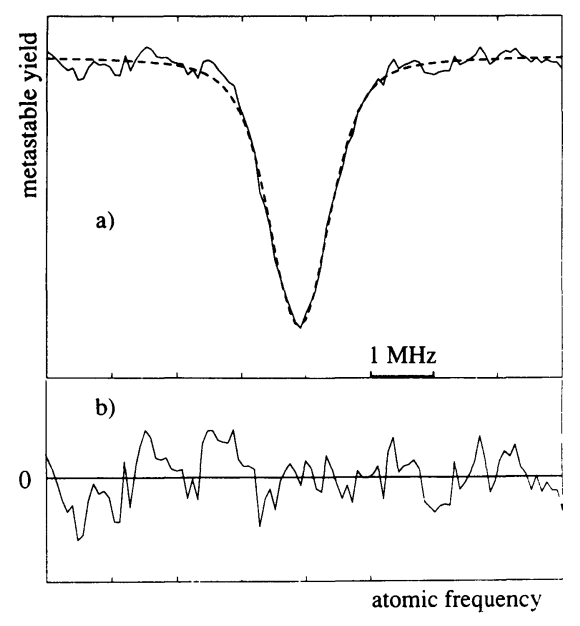

Fig. 8. - a) Fit of the experimental line profile $\left(2 S_{1 / 2}-10 D_{5 / 2}\right.$ in hydrogen) with the calculated one. b) Difference in an expanded scale between the experimental and theoretical curves shown in a). The frequency scale is in terms of the atomic transition frequency.

calibration in order to determine the light power, which is directly connected to the line intensity.

The following procedure is used. We have included all broadening effects not taken into account in the theoretical calculation (i.e. those considered in sections 2.1, 2.2, 2.3, 2.5 and 2.6) by convolution of the theoretical line profile with a Gaussian curve. For each signal recording we can fit the theoretical curve by adjusting four parameters : the metastable yield when the laser is off resonance, the light power $P$, the line position without light-shift (with respect to our very stable reference cavity) and the Gaussian broadening.

As an example, figure 8a shows the fit of an experimental signal relative to the transition $2 \mathrm{~S}_{1 / 2}$ $10 \mathrm{D}_{5 / 2}$ in hydrogen with the theoretical line profile. The difference between the experimental 
profile and the theoretical one is plotted in figure $8 \mathrm{~b}$ which shows that there is no systematic error in the fit.

In order to compare precisely the experimental and theoretical profiles, we have averaged several recordings obtained with the same power so that the signal-to-noise ratio is increased. The best value of $R_{1}$ used in equation (11) has been deduced from such a comparison. This is illustrated by figures $9 \mathrm{a}$ and $9 \mathrm{~b}$ which show the result for $R_{1}=3.5 \mathrm{~mm}$ and $R_{1}=1.5 \mathrm{~mm}$. In the first case there is a systematic effect because the asymmetry of the theoretical curve is too large. In the second case $\left(R_{1}=1.5 \mathrm{~mm}\right)$ this discrepancy disappears and the relative difference between the two curves is much smaller (signal-to-noise ratio $\sim 100$ ). The whole set of our experimental results have been analyzed with the value $R_{1}=1.5 \mathrm{~mm}$. We will give another reason for this choice in 4.3.
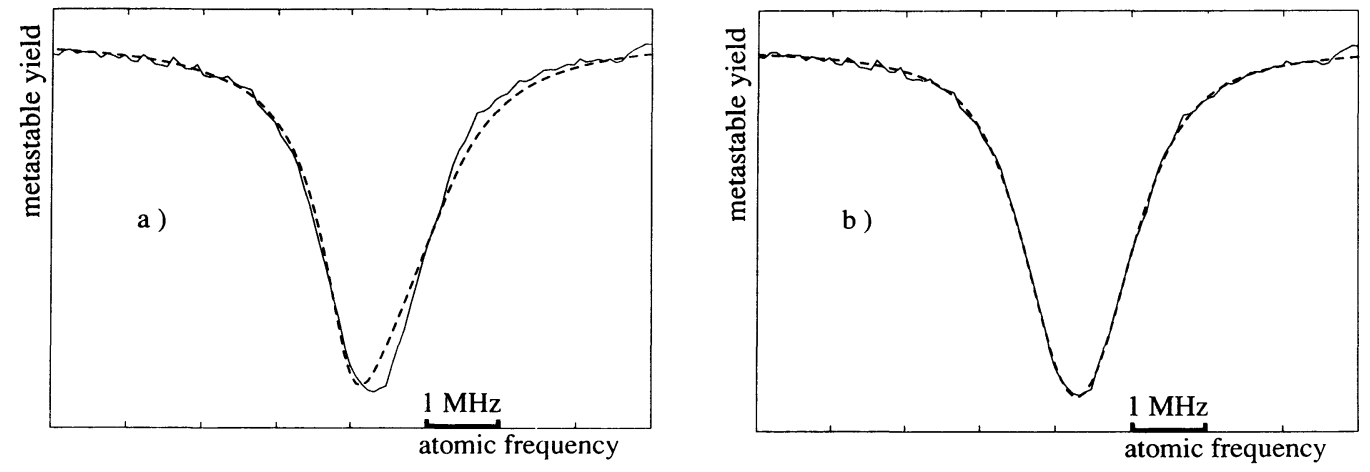

Fig. 9. - Comparison between the experimental curve and the theoretical one, calculated for two $R_{1}$ values : a) $R_{1}=3.5 \mathrm{~mm}$; b) $R_{1}=1.5 \mathrm{~mm}\left(2 \mathrm{~S}_{1 / 2}-8 \mathrm{D}_{5 / 2}\right.$ transition in hydrogen).

In order to determine the Rydberg constant we have studied the three transitions $2 \mathrm{~S}_{1 / 2}-$ $n \mathrm{D}_{5 / 2}(n=8,10,12)$ in hydrogen and deuterium. For each transition, we have recorded 56 experimental line profiles for various laser powers. The fitted values of the light power $P$ and of the light-shift corrected line position CLP give two series of data which we will now analyze.

4.2 LIGHT POWER DETERMINATION. - In our experimental set-up, we can monitor the light power inside the enhancement cavity with a photodiode placed after the end mirror of this cavity. Figure 10 shows the fitted power $(P)$ versus the photodiode signal (IT) in the case of the $2 \mathrm{~S}_{1 / 2}-$ $10 \mathrm{D}_{5 / 2}$ transition. There is a good agreement between these two sets of data. Nevertheless these two quantities ( $P$ and IT) are not exactly in a linear ratio as can be deduced from the best fitted straight line which does not pass through the origin. This stray effect is systematic and shows the limits of our fit procedure. Table III gives the slope of this straight line for the six transitions studied. For a given excited level $(n=8,10,12)$, there is a very good agreement between the values obtained for hydrogen and deuterium although the line intensities are different in these two cases (for deuterium the line intensity is larger than for hydrogen, because of the smaller velocity of deuterium atoms). In table III, the comparison between different excited levels is not significant, because the photodiode signal and the cavity mirror transmission depend on the wavelength. 


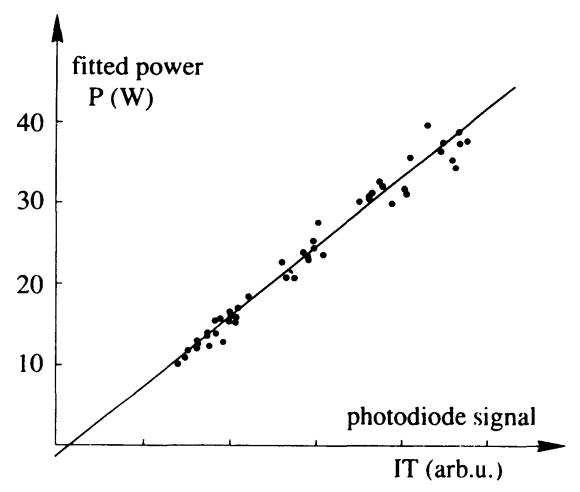

Fig. 10. - Fitted power $P$ versus the photodiode signal IT. Data are relative to 48 recordings of the $2 \mathrm{~S}_{1 / 2}-$ $10 \mathrm{D}_{5 / 2}$ transition in deuterium.

Table III. - Ratio between the fitted power $P$ and the photodiode signal IT (arbitrary units)

\begin{tabular}{|c|c|c|}
\hline & Hydrogen & Deuterium \\
\hline $2 \mathrm{~S}_{1 / 2}-8 \mathrm{D}_{5 / 2}$ & $0.260(10)$ & $0.265(4)$ \\
$2 \mathrm{~S}_{1 / 2}-10 \mathrm{D}_{5 / 2}$ & $0.437(15)$ & $0.443(14)$ \\
$2 \mathrm{~S}_{1 / 2}-12 \mathrm{D}_{5 / 2}$ & $0.44(3)$ & $0.43(2)$ \\
\hline
\end{tabular}

4.3 LINE POSITION. - The light-shift corrected line position (CLP) given by each fit can be compared to the half-maximum center (HMC) of the line. For each transition, CLP has been extrapolated to null light power as shown in figure 11. In this figure, the straight line is least-squares fitted : it is horizontal, showing that the light-shift is well corrected. The observed half-maximum center of the line is also plotted versus the light power : a dotted line has been superimposed given the light-shifted line position calculated from the fitted values of $P$ and CLP. In table IV, the mean light-shift per unit power is compared with the slope of the straight line fitting CLP, for the six transitions studied. The light-shift correction is better than $91 \%$ except for the 2S-12D transition in deuterium. In this case, the disagreement is probably due to the poor signal-to-noise ratio. The atomic frequency is deduced from the CLP extrapolation.

Figure 12 shows the same extrapolation as in figure 11 when the theoretical curves are calculated with $R_{1}=2 \mathrm{~mm}$ (cf. Eq. (11)). The calculated light-shift is too large and we see that the slope of the CLP/P straight line is very sensitive to the $R_{1}$ radius value. For $R_{1}=1.5 \mathrm{~mm}$ this slope is very close to zero. To take into account the nonlinearity between the fitted power $P$ and the photodiode signal IT, we have also made these extrapolations versus the photodiode signal IT. Table $\mathrm{V}$ gives the extrapolation results in these different cases. The first column gives the CLP/P extrapolation uncertainties and the second column the difference between the CLP/IT and CLP/P 


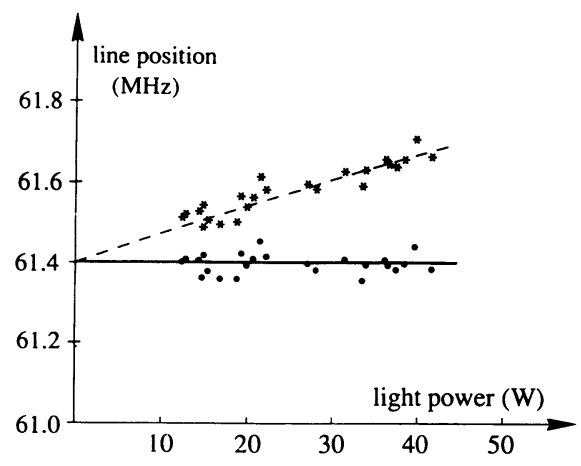

Fig. 11. - Comparison of the light-shifted line position for various light powers (stars) with the corresponding light-shift corrected line position given by the fit (dots) in the case of the $2 S_{1 / 2}-10 D_{5 / 2}$ transition in hydrogen. The straight line is least-squares fitted.

Table IV. - Comparison of the mean light-shift per unit power MLS/P with the slope of the light-shift corrected line position CLP versus the light power.

\begin{tabular}{|c|c|c|}
\hline & $\begin{array}{c}\text { MLS/P } \\
(\mathrm{kHz} / \text { Watt })\end{array}$ & $\begin{array}{c}\text { CLP/P } \\
(\mathrm{kHz} / \text { Watt })\end{array}$ \\
\hline H : 2S - 8D & $5.9(7)$ & $0.5(7)$ \\
2S-10D & $6.0(4)$ & $0.0(4)$ \\
2S-12D & $6.5(8)$ & $0.2(8)$ \\
D : 2S-8D & $5.6(5)$ & $0.4(5)$ \\
$2 S-10 D$ & $5.3(5)$ & $-0.4(5)$ \\
$2 S-12 D$ & $4.7(8)$ & $-1.2(8)$ \\
\hline
\end{tabular}

extrapolations. There is a systematic effect : the mean difference is $-11 \mathbf{k H z}$. For the Rydberg constant determination, we have used the mean of the CLP/P and CLP/IT extrapolations and assumed an error bar including the two values, i.e. an uncertainty of $\pm 8 \mathrm{kHz}$. The third column of table $V$ gives the difference between the CLP/P extrapolation calculated with $R_{1}=2 \mathrm{~mm}$ and with $R_{1}=1.5 \mathrm{~mm}$ : there is a systematic difference, $5 \mathrm{kHz}$ on average, which shows the dependence on $R_{1}$ of the extrapolation. We estimate in this way that the uncertainty due to the choice of the value $1.5 \mathrm{~mm}$ for $R_{1}$ is $5 \mathrm{kHz}$. Our estimate of the total uncertainty due to the extrapolation procedure (henceforth called "uncertainty of the theoretical line shape") is the sum of these two contributions, i.e. $13 \mathrm{kHz}$. The corresponding relative uncertainty is $1.7 \times 10^{-11}$, rounded to $2 \times 10^{-11}$ for the Rydberg constant determination. This uncertainty is different from the statistical one, which is indicated in the first column of table $\mathrm{V}$. 


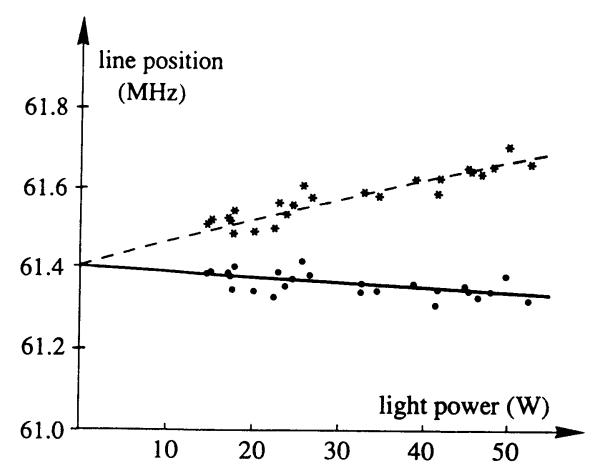

Fig. 12. - Same comparison as that of figure 11, but the theoretical curve is calculated with $R_{1}=2 \mathrm{~mm}$.

Table V. - Results of the corrected line position (CLP) for the six studied transitions. Column 1 lists the uncertainty of CLP vs. light power $(P)$ extrapolation for $R_{1}=1.5 \mathrm{~mm}$. Column 2 gives the differences between the CLP vs. photodiode signal (IT) extrapolation and the CLP vs. P extrapolation. Column 3 gives the difference obtained when CLP vs. $P$ extrapolation is calculated for $R_{1}=2 \mathrm{~mm}$ and $R_{1}=1.5 \mathrm{~mm}$.

\begin{tabular}{|c|c|c|c|}
\hline & $\begin{array}{c}\text { Uncertainty of } \\
\text { CLP/P } \\
\text { extrapolation } \\
R_{1}=1.5 \mathrm{~mm}\end{array}$ & $\begin{array}{c}\text { Difference between } \\
\text { the CLP/IT and } \\
\text { CLP/P extrapolations } \\
R_{1}=1.5 \mathrm{~mm}\end{array}$ & $\begin{array}{c}\text { Difference between } \\
\text { the CLP/P } \\
\text { extrapolations for } \\
R_{1}=2 \mathrm{~mm} \text { and } 1.5 \mathrm{~mm}\end{array}$ \\
\hline $\mathrm{H}: 2 \mathrm{~S}-8 \mathrm{D}$ & $27 \mathrm{kHz}$ & $-20 \mathrm{kHz}$ & $+9 \mathrm{kHz}$ \\
$2 \mathrm{~S}-10 \mathrm{D}$ & $17 \mathrm{kHz}$ & $-6 \mathrm{kHz}$ & $+4 \mathrm{kHz}$ \\
$2 \mathrm{~S}-12 \mathrm{D}$ & $28 \mathrm{kHz}$ & $-15 \mathrm{kHz}$ & $+3 \mathrm{kHz}$ \\
$\mathrm{D}: 2 \mathrm{~S}-8 \mathrm{D}$ & $18 \mathrm{kHz}$ & $+1 \mathrm{kHz}$ & $+4 \mathrm{kHz}$ \\
$2 \mathrm{~S}-10 \mathrm{D}$ & $17 \mathrm{kHz}$ & $-3 \mathrm{kHz}$ & $+3 \mathrm{kHz}$ \\
$2 \mathrm{~S}-12 \mathrm{D}$ & $24 \mathrm{kHz}$ & $-23 \mathrm{kHz}$ & $+6 \mathrm{kHz}$ \\
\hline
\end{tabular}

\section{Conclusion.}

In conclusion, we have carefully analyzed the lineshape of the two-photon transitions. We have performed theoretical calculations of the two-photon transition probability and of the light-shift. The theoretical curves have been compared to the experimental ones and we have precisely described the procedure used to take into account the light-shift. The final result is the determination of the atomic frequencies with respect to our reference cavity with a relative uncertainty of a few parts in $10^{11}$. These determinations will be used in paper III for the Rydberg constant measurement. 


\section{Appendix}

\section{Calculation of the dipole matrix elements.}

We present here the formulae that are used to calculate the matrix elements $R\left(n, \ell ; n^{\prime}, \ell+1\right)=$ $\left\langle n^{\prime}, \ell|r| n, \ell>\right.$ where $| n, \ell>$ and $\left|n^{\prime}, \ell+1\right\rangle$ are discrete states, and their generalization to discrete-continuum coupling [11] :

$$
\begin{array}{r}
R\left(n, \ell ; n^{\prime}, \ell+1\right)=\frac{(-4)^{\ell+1}}{(2 \ell+1) !} z_{2}^{\ell+2} \sqrt{\left(n^{\prime}-\ell-1\right)_{2 \ell+3}(n-\ell)_{2 \ell+1} \times} \\
z_{1}^{n}\left(-z_{1}\right)^{n^{\prime}}\left[F\left(\ell+1-n, \ell+2-n^{\prime} ; 2 \ell+2 ;-4 z_{2}\right)\right. \\
\left.-z_{1}^{2} F\left(\ell+1-n, \ell-n^{\prime} ; 2 \ell+2 ;-4 z_{2}\right)\right]
\end{array}
$$

where: $(a)_{k}=a(a+1) \ldots(a+k-1),(a)_{0}=1$ (Porchamer's symbol)

$F(a, b ; c ; z)=\sum_{k=0}^{\infty} \frac{(a)_{k}(b)_{k}}{(c)_{k}} \frac{z^{k}}{k !}$ (hypergeometric fonction)

(if one of the two levels is a discrete one, the hypergeometric functions are polynomials).

$$
z_{1}=\frac{n-n^{\prime}}{n+n^{\prime}} \text { and } z_{2}=\frac{n n^{\prime}}{\left(n-n^{\prime}\right)^{2}}
$$

(these expressions of $z_{1}$ and $z_{2}$ are useful to calculate $R\left(n, \ell ; n^{\prime}, \ell+1\right)$ for highly excited levels).

Formula (A.1) applies in the case $n \neq n^{\prime}$. For $n=n^{\prime}$, we have:

$$
R(n, \ell ; n, \ell+1)=-\frac{3}{2} n \sqrt{n^{2}-(\ell+1)^{2}}
$$

(which is often written with a wrong sign, that is with a phase definition incompatible with that of (A.1)).

Let us now generalize this formula to the case where one of the levels lies in the continuum. It can be shown [11] that an analytical continuation of equation (A.1) can be made into the continuum by replacing $n$ by $i \eta$ where

$$
\eta=\left(\frac{\mathrm{Ry}}{E}\right)^{1 / 2},\left(\eta=(1 / 2 E)^{1 / 2} \text { in atomic units }\right)
$$

(Ry is the Rydberg constant in energy units and $E$ the energy of the continuum state). This implies a normalization for the continuum states such that the continuum wave functions are related to the discrete ones by:

$$
\Psi_{E, \ell}^{(\text {cont. })}(r)=\frac{\Psi_{i \eta, \ell}^{(\text {bound })}(r)}{\sqrt{\frac{2 i k^{3}}{\eta \pi}[1-\exp (-2 \pi \eta)]}}
$$

where $k=a_{0}^{-1}(E / \mathrm{Ry})^{1 / 2}\left(a_{0}\right.$ is the Bohr radius).

This is in fact equivalent to saying that the bound-continuum matrix elements differ from bound-bound ones, after replacing $n$ by $i \eta$, by a factor $\exp (-i \pi / 4)[1-\exp (-2 \pi \eta)]^{-1 / 2}$; the continuum density of states is then given by:

$$
\rho(E) \mathrm{d} E=(2 E)^{-3 / 2} \mathrm{~d} E \quad \text { (atomic units) }
$$


which is an analytical continuation of the discrete states relation:

$$
\rho(n)|\mathrm{d} n|=1|\mathrm{~d} n|=\rho(E)|\mathrm{d} E|
$$

with $E=-\frac{1}{2 n^{2}}$.

\section{Acknowledgements.}

The authors are indebted to Professor B. Cagnac for many stimulating discussions during this experiment and to Doctor D. Delande for much fruitful advice on the calculation of light-shift and two-photon transition probabilities. This work is partially supported by the Bureau National de Métrologie. One of us (JCG) is grateful to the Brazilian Financial Agency CAPES for a scholarship.

\section{References}

[1] Biraben F., Garreau J.C., JULIEN L. and Allegrini M., Phys. Rev. Lett. 62 (1989) 621.

[2] GarReAu J.C., Allegrini M., Juluen L. and Biraben F., part I., J. Phys. France 51 (1990) 2263

[3] Biraben F., Bassini M. and CAGNAC B., J. Phys. France 40 (1979) 445.

[4] Biraben B., GarReau J.C., Julien L. and Allegrini M., Rev. Sci. Instrum. 61 (1990) 1468.

[5] ROBISCOE R.T. and SHYN T.W., Phys. Rev. Lett. 24 (1970) 559.

[6 BIRABEN F., GARREAU J.C. and JULIEN L., Europhys. Lett. 2 (1986) 925.

[7] CAGNAC B., GRYNBerg G. and BirABEN F., J. Phys. France 34 (1973) 845.

[8] BASSANI F., ForNeY J. and QuaTtropani A., Phys. Rev. Lett. 39 (1977) 1070.

[9] DELANDE D., private communication.

[10] GARREAU J.C., unpublished thesis (Universite Pierre et Marie Curie, Paris) 1989.

[11] KARZAS W.J. and LATTER R., Astrophys. J. Suppl. Ser. 6 (1961) 167.

Cet article a été imprimé avec le Macro Package "Editions de Physique Avril 1990". 